\title{
Bipartite Embeddings of Trees in the Plane
}

\author{
M. Abellanas ${ }^{1}$ J. García ${ }^{2}$ G. Hernández ${ }^{1}$ M. Noy ${ }^{3}$ and P. Ramos ${ }^{4}$
}

1 Facultad de Informática, Univ. Politécnica de Madrid, Boadilla del Monte, 28660

Madrid, SPAIN. E-mail: mavellanas@fi.upm.es, gregorio@fi.upm.es.

2 Escuela Universitaria de Informática, Univ. Politécnica de Madrid, Crta. de

Valencia, km 7, 28031 Madrid, SPAIN. E-mail: jglopez@eui.upm.es.

3 Dep. de Matemàtica Aplicada II, Univ. Politècnica de Catalunya, Pau Gargallo 5, 08028 Barcelona, SPAIN. E-mail: noy@ma2.upc.es.

4 Escuela Universitaria de I.T. Aeronáutica, Univ. Politécnica de Madrid, Pza.

Cardenal Cisneros s/n, 28040 Madrid, SPAIN. E-mail: pramos@fi.upm.es.

\begin{abstract}
Given a tree $T$ on $n$ vertices and a set $P$ of $n$ points in the plane in general position, it is known that $T$ can be straight line embedded in $P$ without crossings. Now imagine the set $P$ is partitioned into two disjoint subsets $R$ and $B$, and we ask for an embedding of $T$ in $P$ without crossings and with the property that all edges join a point in $R$ (red) and a point in $B$ (blue). In this case we say that $T$ admits a bipartite embedding with respect to the bipartition $(R, B)$. Examples show that the problem in its full generality is not solvable. In view of this fact we consider several embedding problems and study for which bipartitions they can be solved. We present several results that are valid for any bipartition $(R, B)$ in general position, and some other results that hold for particular configurations of points.
\end{abstract}

\section{Introduction}

Given a tree $T$ on $n$ vertices and a set $P$ of $n$ points in the plane in general position, it is known that $T$ can be straight line embedded in $P$ without crossings. The problem becomes more difficult if $T$ is rooted and we want to root it at any particular point of $P$. The problem in this form was posed by Perles and partially solved by Pach and Torosick [6]. A complete solution was found by Ikebe et al. [5]. A related result by A. Tamura and $Y$. Tamura [7] is that, given a point set $P=\left\{p_{1}, \ldots, p_{n}\right\}$ and a sequence $d=\left(d_{1}, \ldots d_{n}\right)$ of positive integers with $\sum d_{i}=2 n-2$, there exists an embedding of some tree in $P$ such that the degree of $p_{i}$ is equal to $d_{i}$. Optimal algorithms for solving the above problems have been found by Bose, McAllister and Snoeyink [2].

In this paper we consider the following embedding problem. A point set $P$ in the plane in general position (no three points collinear) is partitioned into two disjoint sets $R$ and $B$ (the red and the blue points), and we are asked to embed a tree $T$ in $P$ without crossings and with the additional property that all the edges are red/blue, i.e. all edges connect a point in $R$ to another point in $B$. We call such an embedding a bipartite embedding of $T$ with respect to the bipartition $(R, B)$. 
Any tree $T$ is a bipartite graph and the bipartition $V(T)=\left(V_{1}, V_{2}\right)$ induced in the vertex set is in fact unique. An obvious necessary condition for the existence of a bipartite embedding of $T$ in $P$ is that the cardinalities of both bipartitions, those of $T$ and of $P$, match correctly. However, simple examples show that this is not always sufficient. It is then natural to relax the requirements of the problem and to ask: given a bipartition $(R, B)$, is it always possible to find a bipartite embedding of some tree with respect to $(R, B)$ ? It is straightforward to prove that the answer is affirmative. Take any red point and join it to all the blue points. It is then clear that the remaining red points can be connected to suitable blue points wihtout creating crossings. This simple solution has the shortcoming of producing trees with very large maximum degree.

The approach taken in this paper is to consider several natural embedding problems and to investigate for which bipartitions they can be solved. The problems we study (considering $(R, B)$ as the input) are:

1) find a bipartite embedding of a tree with bounded degree;

2) find a bipartite embedding of a tree where the degrees of the vertices in one of the parts, say $R$, are prescribed in advance;

3) find a bipartite embedding of a spanning path.

The paper is organized as follows. In Section 2 we discuss in detail a collection of problems about bipartite embeddings. Section 3 contains the results for points in general position, while in Section 4 we restrict our attention to particular configurations of points. We conclude with a brief discussion of some open problems.

\section{Discussion of the problems}

In this section we fix the terminology we use in the remainder of the paper, and we state and discuss in detail three embedding problems.

$P$ denotes a set of $n$ points in the plane in general position (no three of them collinear), partitioned into two subsets $R=\left\{p_{1}, \ldots, p_{r}\right\}$ and $B=\left\{q_{1}, \ldots, q_{b}\right\}$ with cardinalities $r=|R|$ and $b=|B|$. The bipartition is denoted $(R, B)$. The set of vertices in the convex hull of $P$ is denoted $C H(P)$.

Let $T$ be a spanning tree of the complete bipartite graph $K_{r, b}$, and let $\left(d_{1}, \ldots, d_{r}\right)$ and $\left(d_{1}^{\prime}, \ldots, d_{b}^{\prime}\right)$ be the degree sequences corresponding to the two parts of the bipartition. Since the number of edges in $T$ is $r+b-1$ we have that

$$
\sum_{i=1}^{r} d_{i}=\sum_{j=1}^{b} d_{j}^{\prime}=r+b-1 .
$$

Given a tree $T$ on $n$ vertices, we say that $T$ admits a bipartite embedding with respect to $(R, B)$ if $T$ can be straight-line embedded into $P$ without crossings, i.e. edges can only intersect at the vertices, and with the additional property that an edge always joins a point in $R$ and a point in $B$. An obvious necessary 
condition for the existence of such an embedding is that $T$ is a spanning tree of $K_{r, b}$. In other words, if $V(T)=V_{1} \cup V_{2}$ is the unique bipartition of the vertex set of $T$, then we must have either $\left|V_{1}\right|=r,\left|V_{2}\right|=b$ or $\left|V_{1}\right|=b,\left|V_{2}\right|=r$.

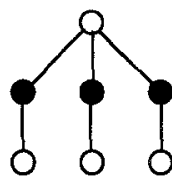

$\mathrm{T}$

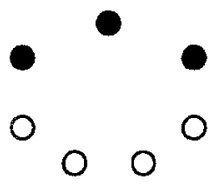

$(\mathrm{R}, \mathrm{B})$

Fig. 1. $T$ does not admit a bipartite embedding with respect to $(R, B)$.

This condition is not always sufficient, as the example in Figure 1 shows. Because of this fact we adopt the following point of view. The bipartition $(R, B)$ is given as input, and the problem is to find a bipartite embedding of some tree with respect to $(R, B)$ satisfying a given condition. We introduce three conditions that give rise to the three problems discussed below.

Bounded degree embeddings. Let $T$ be a spanning tree of $K_{r, b}$, and let $d_{1}, \ldots, d_{r}$ and $d_{1}^{\prime}, \ldots, d_{b}^{\prime}$ be the degree sequences of the vertices of the two parts. The equation $\sum d_{i}=r+b-1$ implies that $T$ has a vertex whose degree is at least $1+\lceil(b-1) / r\rceil$ (this is the red mean degree). Our first problem asks for the existence of a bipartite embedding attaining a bound of this order of magnitude (we show later that the exact bound cannot always be achieved).

Problem 1. Given a bipartition $(R, B)$ with $r \leq b$, find a bipartite embedding of a tree with respect to $(R, B)$ having maximum degree $\Delta=O(b / r)$.

Fixed degree embeddings. Let $\mathbf{d}=\left(d_{1}, \ldots, d_{r}\right)$ and $\mathbf{d}^{\prime}=\left(d_{1}^{\prime}, \ldots, d_{b}^{\prime}\right)$ be sequences of positive integers satisfying $\sum d_{i}=\sum d_{j}^{\prime}=r+b-1$. It is easy to see that $K_{r, b}$ admits a spanning tree whose degree sequences are equal to $\mathbf{d}$ and $\mathbf{d}^{\prime}$, respectively. However, given $(R, B)$ it is not always possible to find a bipartite embedding realizing both degree sequences (take $\mathbf{d}=(2,2,2)$ and $\mathbf{d}^{\prime}=(3,1,1,1)$ in the example of Figure 1). Our second problem asks for a bipartite embedding in which the degree of every vertex in $R$ is fixed in advance.

Problem 2. Given a bipartition $(R, B), R=\left\{p_{1}, \ldots, p_{r}\right\}$, and a sequence of positive integers $\left(d_{1}, \ldots, d_{r}\right)$ with $\sum d_{i}=r+b-1$, find a bipartite embedding of a tree with respect to $(R, B)$ such that the degree of $p_{i}$ is $d_{i}$.

Embedding a spanning path. If we take $\Delta=2$ in Problem 1 then we are asking for a bipartite embedding of a spanning path. An obvious necessary condition 
is $|r-b| \leq 1$. The example in Figure 2 shows that it is not sufficient. To see why note that the first edge of the path has to be an edge of the convex hull. The fact that the cardinalities of consecutive red and blue chains differ always in more than two units, prevents the path from spanning all the vertices. Our last problem asks for sufficient conditions that guarantee the existence of such a path.

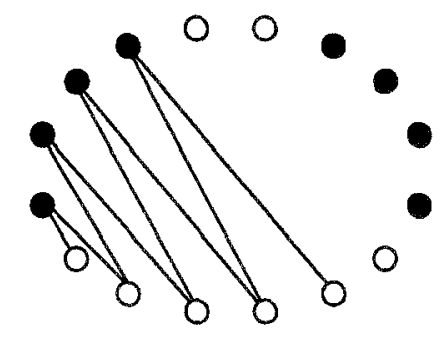

Fig. 2. The bipartite path cannot be completed to a spanning path.

Problem 3. Characterize those bipartitions $(R, B)$ with $|r-b| \leq 1$ admitting a bipartite embedding of a spanning path.

We find a complete solution to Problem 2, and prove several results concerning Problems 1 and 3. These are presented in the next two sections.

\section{Points in general position}

In this section we present two results that hold for any bipartition in general position. The first one provides an answer to Problem 1 and the second one solves completely Problem 2.

Theorem 4. Given a bipartition $(R, B)$ in the plane with $r \leq b$, one can find a bipartite embedding of a tree with respect to $(R, B)$ such that the maximum degree $\Delta$ is $O(b / r+\log r)$.

Proof. We use the ham-sandwich theorem [3], that asserts that the sets $R$ and $B$ can be simultaneously bisected by a straight line. Assume for simplicity that $r=2^{k}$ and that $b=\alpha r=\alpha 2^{k}$. Applying repeatedly the ham-sandwich theorem we arrive, after $k$ steps, to a partition of the plane into convex polygonal regions, each one of them containing exactly one red point and $\alpha$ blue points. Join every red point to the corresponding $\alpha$ blue points to obtain a collection of $r$ disjoint copies of a bipartite embedding of a star $K_{1, r}$. 
Next we merge these $r$ partial trees into a single tree, in the opposite order as they have been produced, preserving the bipartite character of the embedding. To do that we need the following lemma, whose easy proof is omitted.

Lemma. Given two disjoint bipartite embeddings $T_{1}$ and $T_{2}$ separated by a straight line, one can add an additional edge between $T_{1}$ and $T_{2}$, resulting in a global bipartite embedding of a tree.

We have to perform $k$ merging steps, every time reducing by half the number of trees. It is clear that at every step the maximum degree increases at most by one. Since initially $\Delta=\alpha$, at the end $\Delta \leq \alpha+k=b / r+\log _{2} r$. The cases where $r$ is not a power of two or $b / r$ is not an integer are treated similarly.

In the next section it will be shown that the optimal bound $O(b / r)$ can be achieved for several particular configurations.

Theorem 5. Given a bipartition $(R, B)$ in the plane, with $R=\left\{p_{1}, \ldots, p_{r}\right\}$ and a sequence $\left(d_{1}, \ldots, d_{r}\right)$ of positive integers with $\sum d_{i}=r+b-1$, there exists a bipartite embedding of a tree with respect to $(R, B)$ such that the degree of $p_{i}$ is equal to $d_{i}$.

Proof. The proof is by induction on $r+b$. Assume without loss of generality that $d_{1}=\max d_{i}$, and let $l$ be an oriented line through $p_{1}$ not containing any other point from $R$ or $B$. Let $H^{+}$and $H^{-}$be the right and left open halfspaces in which the line $l$ divides the plane. Let $r^{+}=\left|R \cap H^{+}\right|$and $b^{+}=\left|B \cap H^{+}\right|$, and let also $r^{-}=\left|R \cap H^{-}\right|$and $b^{-}=\left|B \cap H^{-}\right|$. Finally, define two functions (that depend on $l) f^{+}$and $f^{-}$as follows

$$
\begin{aligned}
& f^{+}=\sum_{p, \in H^{+}} d_{i}-r^{+}-b^{+} \\
& f^{-}=\sum_{p, \in H^{-}} d_{i}-r^{-}-b^{-},
\end{aligned}
$$

and observe that $f^{+}+f^{-}=-d_{1}$.

We claim that there exists some position of $l$ in which $-d_{1}<f^{+}<0$. To prove the claim assume that initially $f^{+} \geq 0$ and consider the changes in $f^{+}$as $l$ turns around $p_{1}$.

If a red point $p_{i}$ enters $H^{+}$, then $f^{+}$increases by $d_{i}-1$;

If a red point $p_{i}$ exits $H^{+}$, then $f^{+}$decreases by $d_{i}-1$;

If a blue point enters $H^{+}$, then $f^{+}$decreases by 1 ;

If a blue point exits $H^{+}$, then $f^{+}$increases by 1 .

In any case the change in absolute value is at most $d_{i}-1 \leq d_{1}-1$. Since after a turn of 180 degrees the values of $f^{+}$and $f^{-}$are interchanged, and $f^{+}=-d_{1}-f^{-}$, it follows that $f^{+} \leq-d_{1}$. All this implies that for some intermediate value we 
have $-d_{1}<f^{+}<0$. If we assume instead that initially $f^{+} \leq-d_{1}$ we proceed in the same way, and the claim is proved.

Now by induction we can find a bipartite embedding of a tree with respect to the bipartition $\left(\left(R \cap H^{+}\right) \cup\left\{p_{1}\right\}, B \cap H^{+}\right)$in which $p_{1}$ has degree $-f^{+}$and $p_{i}$ has degree $d_{i}$ for $p_{i} \in R \cap H^{+}$. Similarly we get a tree on $H^{-}$in which $p_{1}$ has degree $-f^{-}$, and the union of the two trees does the job.

\section{Points in restricted positions}

We have already mentioned that a bipartition does not always admit a spanning path, and that Theorem 4 does not give the best possible bound for the maximum degree. It is then natural to restrict the geometry of the problem in order to obtain positive results. We consider three such restrictions, or particular positions, that arise naturally. Firstly, when $R$ and $B$ are separated by a straight line. Secondly, when $R \cup B$ is a set in convex position. And finally when the vertices of $R$ define a convex polygon containing all the vertices in $B$.

\subsection{Linearly separable partitions}

We say that a bipartition $(R, B)$ is linearly separable if there exists a straight line separating $R$ and $B$. Equivalently, if the convex hulls of $R$ and $B$ are disjoint.

Theorem 6. Every linearly separable bipartition $P=R \cup B$ with $|r-b| \leq 1$ admits a bipartite embedding of a spanning path.

Proof. Assume without loss of generality that $R$ and $B$ are separated by a horizontal line, and let $p_{1} \in R$ and $q_{1} \in B$ be such that $p_{1} q_{1}$ is the left red/blue edge of the convex hull of $P$. We say that $p_{1} q_{1}$ is the left bridge of $P$. The initial point of the spanning path will be $p_{1}$ if $b=r-1, q_{1}$ if $b=r+1$, and either $p_{1}$ or $q_{1}$ if $b=r$. Assume we start at $p_{1}$ and set $C=\left\{p_{1}\right\}(C$ is an ordered list that corresponds to the spanning path as it is constructed). At every step compute the left bridge $p q$ of $P \backslash C$, and add $p$ to $C$ if the last point in $C$ is in $B$, or add $q$ if the last point in $C$ is in $R$. In this way we get a bipartite embedding of a path that has no crossings because $C$ is disjoint from the convex hull of $P \backslash C$ and hence from all edges added to it during the algorithm.

The technique in the above proof can also be used to prove the following result.

Theorem 7. Every linearly separable bipartition $P=R \cup B$ with $r \leq b$ admits a bipartite embedding of a tree $T$ with $\Delta(T) \leq 1+[(b-1) / r\rceil$.

Proof. Let $\bar{d}=1+\lceil(b-1) / r\rceil$. The idea is to find a bipartite embedding of a tree with respect to $(R, B)$ in which the degrees in $R$ are equal to $\bar{d}$ or to $\bar{d}-1$ and, at the same time, be able to bound the degrees in $B$. 
First we can suppose that $\vec{d}>2$, that is $b-1>r$. Otherwise, since we are assuming $b \geq r$, Theorem 6 implies the existence of a bipartite embedding with $\Delta=2$ and the theorem holds. Now find a sequence $\left(d_{1}, \ldots, d_{r}\right)$ of positive integers such that $\sum d_{i}=r+b-1$ and $d_{i}=\bar{d}$ or $d_{i}=\bar{d}-1$. We could use Theorem 5 in order to find a bipartite embedding realizing this degree sequence, but then we would not be able to control the degrees in $B$.

Instead we proceed as follows. Find a point $p_{1}$ in $R$ such that there is a line separating $p_{1}$ and $d_{1}$ points $q_{1}, \ldots, q_{d_{1}}$ in $B$ from the remaining points, where the $q_{j}$ are sorted in polar order with respecto to $p_{1}$. This is always possible taking left bridges as in the proof of the previous theorem. Join $p_{1}$ to $q_{1}, \ldots, q_{d_{1}}$, remove all these points except $q_{d_{1}}$ from the bipartition and find a new point $p_{2}$ in $R$ that can be separated together with to $d_{2}$ points in $B$. If we repeat this process, at the end we get a single point $p_{r}$ in $R$ and $d_{r}$ points in $B$ (see Figure 3 for an illustration). The fact that $d_{i}>1$ for every $i$, implies that the degrees of the points in $B$ are equal to one or two.

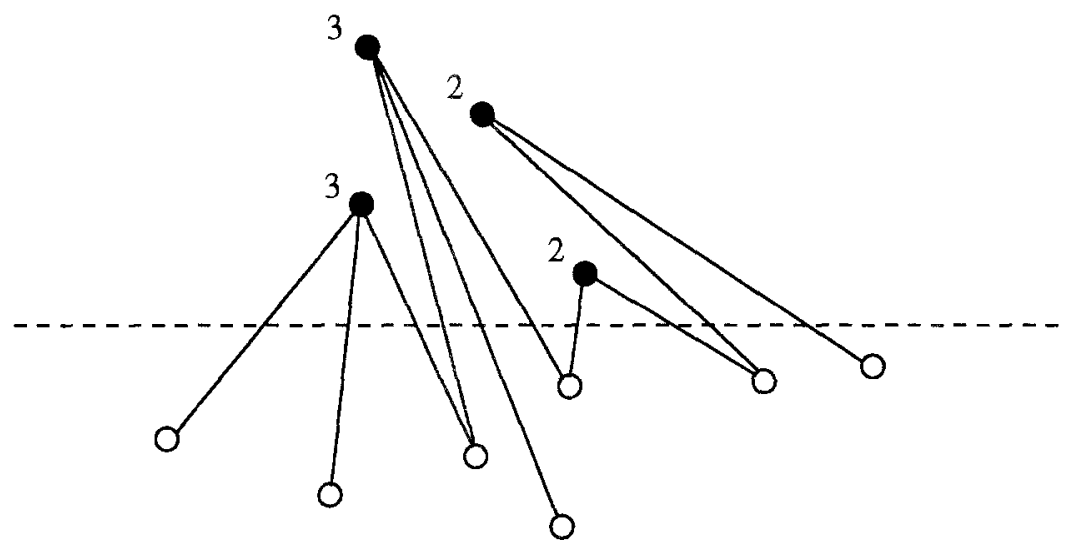

Fig. 3. Realizing the degree sequence $(3,3,2,2)$.

\subsection{Sets in convex position}

We say that a set of points is in convex position if it is the vertex set of a convex polygon. We have already seen an example of a bipartition in convex position with $|r-b| \leq 1$ which does not admit a spanning path, and the example can be generalized to any number of points (larger than 15). On the positive side we prove that Problem 1 can be solved in this case. If $R \cup B$ is in convex position and $r=b$, Problem 3 was solved by Akiyama and Urrutia [1]. They gave an algorithm that determines if $R \cup B$ admits a spanning path in $O\left(n^{2}\right)$ time finding such path if it exists. 
Theorem 8. Let $R \cup B$ be a set in convex position with $r \leq b$. Then the bipartition $(R, B)$ admits a bipartite embedding of a tree $T$ with $\Delta(T) \leq\lceil b / r\rceil+2$.

Proof. Assume for simplicity that $r=2^{k}$ and that $b=\alpha 2^{k}$. Using ham-sandwich cuts as in the proof of Theorem 4 we can obtain $r$ disjoint red/blue copies of a star $K_{1, \alpha}$. The key point in this case is that we can control the degrees in the merging step.

Set initially $T$ equal to any of the $r$ stars. For every edge $e$ of the convex hull of $T$ that is not an edge of the convex hull of $R \cup B$, consider the trees $T_{1}, \ldots, T_{j}$ that are visible from $e$ and lie on the halfspace determined by $e$ not containing $T$, ordered clockwise (this makes sense because the set is in convex position).

Next select one of the vertices of $e$ and construct a bipartite polygonal chain connecting $T$ and the trees $T_{1}, \ldots, T_{j}$ (see Figure 4). Because the set is in convex position, we can construct this chain in such a way that the degree of any vertex increases by at most 2 . Set $T$ equal to the tree obtained with the above costruction and iterate the process until $T$ is a spanning tree.

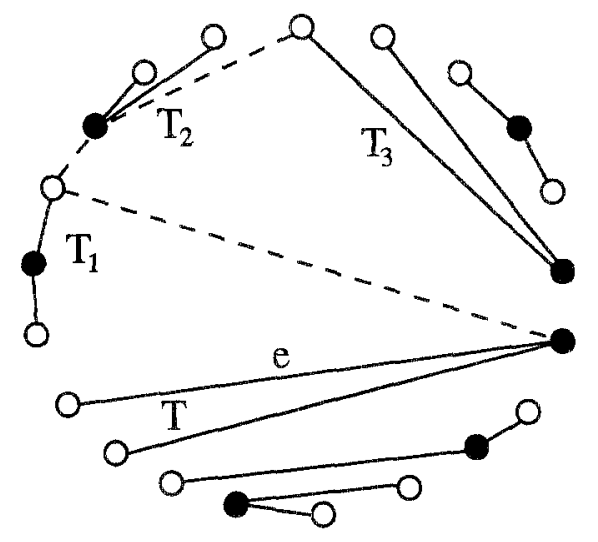

Fig. 4. Proof of Theorem 4.3.

To see that the condition on the degree is satisfied, observe that the new edges of $C H(T)$ that are not in $C H(R \cup B)$ are determined by two vertices that belong to one of the trees $T_{1}, \ldots, T_{j}$. Since only one vertex of each tree is used when constructing the polygonal chain, we can guarantee that we always have a free vertex to iterate the process. Therefore, points in $R$ have degree at most $\alpha+2=b / r+2$, and points in $B$ have degree at most 3 .

\subsection{Bipartitions in which $R=C H(R \cup B)$}

The situation can be described in this way: the points of $R$ are the vertices of a convex polygon containing the points of $B$. We need the following result by García and Tejel [4]. 
Lemma 9. Let $P$ be a set of points in general position, and assume that $C H(P)=$ $\left\{p_{1}, \ldots, p_{k}\right\}$ and that there are $n$ interior points. Let $n=n_{1}+\ldots+n_{k}$, where the $n_{i}$ are positive integers. Then the convex hull of $P$ can be partitioned into $k$ convex polygons $Q_{1}, \ldots, Q_{k}$ such that $Q_{i}$ contains $n_{i}$ points and $p_{i} p_{i+1}$ is an edge of $Q_{i}$.

Proof. (Sketch of the proof by García and Tejel) The proof is by induction on $k$. If $k=3$, by continuity arguments and due to the generic position of points, it is easy to prove that it exists a point $q \notin P$ inside the triangle $p_{1} p_{2} p_{3}$ such that triangles $p_{1} p_{2} q, p_{2} p_{3} q$ and $p_{3} p_{1} q$ contains $n_{1}, n_{2}, n_{3}$ points respectively.

The induction step begin by considering an arbitrary diagonal, for example $p_{1} p_{j}, 1<j<k$. Without loss of generality one can asume that the polygon $p_{1}, \ldots, p_{j}$ contains $n_{1}+\ldots+n_{j-1}$ or more points. In this polygon we can apply the induction hypothesis. Let $p_{1}, q_{1}, \ldots, q_{i}, p_{j}$ the polygon obtained in the previous decomposition corresponding to the edge $p_{1} p_{j}$. If this polygon contains $n_{j}$ or more points, connecting $p_{j+1}$ with a point $q^{\prime}$ in the polygonal chain $p_{1}, q_{1}, \ldots, q_{i}, p_{j}$ the polygon splits into two parts satisfying the induction hypothesis. In other case it is the polygon $p_{1}, p_{j+1}, \ldots, p_{k}$ which verify such condition.

Using the above lemma it is not difficult to prove the following results.

Theorem 10. Let $(R, B)$ be a bipartition in which $R=C H(R \cup B)$ and with $r \leq$ $b$. Then it admits a bipartite embedding of a tree $T$ with $\Delta(T) \leq 1+\lceil(b-1) / r\rceil$.

Proof. If $b=r \alpha+1$ we can use lemma 4.5 to decompose the convex hull of $R$ into $r$ convex polygons $Q_{1}, \ldots, Q_{r}$ such that $Q_{j}, j \neq 1$, contains $\alpha$ points of $B$ , $Q_{1}$ contains $\alpha+1$ points and $p_{i} p_{i+1}$ is an edge of $Q_{i}$.

Let $s_{j} \in Q_{j} \cap B$ such that $\operatorname{dist}\left(s_{j}, p_{j}\right)=\min \left\{\operatorname{dist}\left(s, p_{j}\right): \quad s \in Q_{j} \cap B\right\}$. We join every red point $p_{j}$ to the corresponding $\alpha$ blue points in $Q_{j-1}$ except $s_{1}$. Next we merge these $r$ partial trees, by connecting $s_{j}$ to $p_{j}$. In this way we obtain a tree $T$ with $\Delta(T)=1+\alpha$.

If $\frac{b-1}{r}$ is not an integer then we obtain a tree with $\Delta(T) \leq 1+\left\lceil\frac{b-1}{r}\right\rceil$.

Theorem 11. Let $(R, B)$ be a bipartition with $R=C H(R \cup B)$ and $|r-b| \leq 1$. Then it admits a bipartite embedding of a spanning path.

Proof. If $r=b$, in the partition of the lemma 4.5 every $Q_{j}$ contains only one blue point. Hence it is easy to obtain a bipartite embedding of a spanning path. If $|r-b|=1$ we proceed in a similar way.

\section{Concluding remarks}

In this paper we have introduced the problem of finding bipartite embeddings of trees in the plane and have obtained a number of results. Some of our results are valid for any set of points in general position, and some of them only apply 
to particular configurations of points. There are several interesting questions left open. One is whether one can always achieve the optimal bound in Problem 1, that is, whether any bipartition $(R, B)$ with $r \leq b$ admits a bipartite embedding of a tree with degree bounded by $O(b / r)$. Finally, we remark that most of our proofs are constructive and actually provide algorithms for solving the various problems. A direct analysis of the proofs shows, for example, that the construction in Theorem 3.2 can be done in $O\left(n^{2} \log n\right)$ time, where $n=r+b$, and those in Theorems 4.1 and 4.2 can be done in time $O\left(n \log ^{2} n\right)$. We leave as an open question to find optimal algorithms for these constructions.

\section{Acknowledgements}

Thanks are due to Sue Withesides, Jorge Urrutia and to the referees for their suggestions which contributed to the final version.

\section{References}

1. J. Akiyama and J. Urrutia, Simple Alternating Path Problems, Discrete Mathematics 84 (1990), pp. 101-103.

2. P. Bose, M. McAllister and J. Snoeyink, Optimal Algorithms to Embed Trees in the Plane, in Proc. Graph Drawing 95, Springer Verlag LNCS Vol. 1027, pp.64-75.

3. H. Edelsbrunner, Algorithms in Combinatorial Geometry, Springer Verlag (Berlin, 1987).

4. A. García and J. Tejel, Dividiendo una nube de puntos en regiones convexas, Actas VI Encuentros de Geometria Computacional, pp. 169-174, 1995.

5. Y. Ikebe, M. Perles, A. Tamura and S. Tokunaga, The Rooted Tree Embedding Problem into Points in the Plane, Discrete and Computational Geometry 11 (1994), pp. 51-63.

6. J. Pach and J. Töröcsik, Layout of Rooted trees, in Planar Graphs (W.T. Trotter, ed.), DIMACS Series, Vol. 9, Amer. Math. Soc., pp. 131-137.

7. A. Tamura and Y. Tamura, Degree Constrained Tree Embedding Into Points in the Plane, Information Proc. Letters 44 (1992), pp. 211-214. 